\title{
Effect of polysaccharide emulsifiers on the fabrication of monodisperse oil-in-water emulsions using the microchannel emulsification method
}

\begin{abstract}
The microchannel emulsification method was used to prepare monodisperse soybean oil-inwater emulsions, with polysaccharides as the sole emulsifier. The effects of different types (sodium alginate, carboxymethyl cellulose, pectin, gum arabic) and concentrations $(0.1,0.5$, $1.0 \%$ ) of polysaccharide on the droplet size, droplet size distribution and stability of the emulsions were investigated. The droplet diameters of the resulting emulsions were in the range of $35 \pm 2$ to $47 \pm 3 \mu \mathrm{m}$, with coefficient of variations $(\mathrm{CV})$ of below $6.6 \%$. Generally, the droplet size increased as the concentration of the polysaccharides increased. The stability of emulsions prepared using $0.5 \%$ of sodium alginate, carboxymethyl cellulose and gum arabic was also evaluated in situ. Sodium alginate- and gum arabic-stabilized emulsions were stable for at least $6 \mathrm{~h}$.
\end{abstract}

Keyword: Droplet formation; Low-energy method; Natural emulsifier; Spontaneous; Stabilizer; Uniform droplet size 